\section{Repetition-based Training for Efficient Propulsion in New Manual Wheelchair Users}

\section{Kristin Will', Jack R Engsberg ${ }^{1}$, Matthew Foreman' ${ }^{1}$, Joseph Klaesner ${ }^{2}$, Rebecca Birkenmeier ${ }^{1,2}$ and Kerri Morgan ${ }^{1,2 *}$}

${ }^{1}$ Program in Occupational Therapy, Washington University School of Medicine, St. Louis, Missouri, USA

${ }^{2}$ Program in Physical Therapy, Washington University School of Medicine, St. Louis, Missouri, USA

\begin{abstract}
Objective: To determine the number of propulsion repetitions necessary to produce positive changes in propulsion biomechanics of new manual wheelchair users with spinal cord injuries.

Design: Single subject design.

Setting: Community-based research facility.

Participants and intervention: Five new manual wheelchair users with a spinal cord injury (3 men, 2 women, aged 39.0 \pm 30 ) participated in a nine-session manual wheelchair training program that aimed to improve propulsion biomechanics through 5,400 propulsion repetitions.

Outcome measures: Assessments were performed on a wheelchair dynamometer at seven levels of repetition dosing Kinematic measurements (i.e., push loop height, push angles, cadence) were taken using video cameras and Microsoft Kinect systems. Kinetic measurements (i.e., peak force, average force, force rate of rise) were taken using a wheelchair dynamometer system.

Result: Each of the five participants had improvements in their propulsion biomechanics (e.g., three participants increased their push angle), which occurred in the first levels of repetition dosing (between 1000-2700 repetitions); there were variances in type of change (kinematic or kinetic).
\end{abstract}

Conclusion: Results suggest that proper propulsion biomechanics can be learned with appropriate dosing. The variability among participants in the type of change that occurred at different dosing levels may be due to differences in wheelchair positioning and level of injury. The impact of manual wheelchair users learning

${ }^{*}$ Corresponding author: Kerri Morgan, Program in Occupational Therapy Washington University School of Medicine, Campus Box 8505, 4444 Forest Park Blvd, St. Louis, MO 63108, USA, Tel: +1 3142861659; E-mail: morgank@wusm.wustl.edu

Citation: Will K, Engsberg JR, Foreman M, Klaesner J, Birkenmeier R, et al. (2015) Repetition-Based Training for Efficient Propulsion in New Manual Wheelchair Users. J Phys Med Rehabil Disabil 1: 001.

Received: July 13, 2015; Accepted: August 28, 2015; Published: September 18, 2015 efficient propulsion is great, as engagement in daily activities is dependent upon the health of the upper extremities.

Keywords: Manual wheelchair users; Propulsion; Repetition training; Spinal cord injury; Upper extremity

\section{Introduction}

Many individuals with a Spinal Cord Injury (SCI) depend on the use of manual wheelchairs for mobility and for participation in activities such as personal care, transportation, and community life. Manual wheelchair propulsion requires repetitive motion of the upper extremities, which increases the level of stress on muscles and joints and causes pain that leads to overuse injuries (eg: rotator cuff injuries, tendonitis, carpal tunnel syndrome, and median nerve injuries) [1-4]. Upper extremity pain and injury are major concerns for Manual Wheelchair Users (MWU) due to their dependence on the upper extremities for activities of daily life such as wheelchair propulsion, personal care, dressing, and transfers [5]. Chronic overuse injuries are associated with improper biomechanics and poor ergonomics during wheelchair propulsion $[1,3,6,7]$. As a result of the high number of MWU who experience pain and overuse injuries in their upper extremities, the Consortium for Spinal Cord Medicine (CSCM) developed clinical practice guidelines to reduce the mechanical load on the upper extremities and lessen the chances of developing chronic overuse injuries [8]. These recommendations include minimizing the force applied to the pushrim (push force) and maximizing the push length on the pushrim (push angle) during the push phase of propulsion, decreasing overall push cadence, and dropping the hand below the pushrim toward the axle during the recovery phase [8]

However, since the clinical practice guidelines were developed, evidence based training programs have not been introduced into clinical practice, and many new MWU receive little to no instruction on how to properly propel their chairs $[6,8,9]$. Evidence-based training programs that have been implemented introduce strengthening and/or propulsion techniques as a means to improve wheelchair efficiency but have demonstrated little success with the retention of the acquired techniques, leaving the MWU at greater risk for chronic overuse injuries $[10,11]$. Further, the majority of these training programs did not focus on prevention but remediation and compensation after the onset of the injury $[10,11]$. One pilot study aimed to address prevention of chronic overuse injuries by educating participants on the concepts of the clinical practice guidelines during the acute care phases of rehabilitation following SCI [12]. This was the first study we are aware of that implemented the clinical practice guidelines with new MWU to prevent secondary injuries rather than remediation after the onset of symptoms.

Contemporary motor learning theory suggests that MWU have the potential to learn proper propulsion biomechanics if a sufficient number of repetitions is achieved during training [13]. An important aspect in the development of training programs is understanding when neuroplastic changes occur in order to learn and retain new motor skills $[14,15]$. The long-term retention of 
motor skills plays an important role in everyday activities as it allows for quick and accurate execution of movement. Cortical neuroplastic changes occur following the acquisition of a novel motor skill and "stand to provide the greatest potential for rehabilitation success" [14]. Current research on motor learning suggests that one must perform hundreds of task-specific repetitions over several sessions in order for these neuroplastic changes to occur and be retained [14,16-18]. Repetition-based training has been used successfully in the rehabilitation of other populations with significant health conditions such as stroke [13-16] and Parkinson's Disease $[19,20]$. Task-specific training research for individuals with SCIs has been limited to animal studies [21,22] and gait training $[23,24]$. Studies have shown that significant anatomical changes occur to the sensorimotor system following SCI $[25,26]$ and that these changes may increase upper extremity feedback to the sensorimotor cortex of the brain [26]. To our knowledge, repetition-based training has not been used in propulsion training of new MWU with SCIs.

Currently, there is limited evidence related to acquiring novel motor skills in individuals learning to propel a manual wheelchair following an SCI [12]. By investigating components of training sessions such as optimal duration of training, the level of dosing repetitions required for retention, and training techniques for teaching propulsion, we can identify training parameters that promote efficient skill acquisition in MWU. Clinical rehabilitation literature identifies small sample single subject design studies as beneficial in piloting rehabilitation interventions, and they are increasingly used [27-29]. While single subject design methods are unable to conclude the generalized efficacy of treatments, they permit investigators to identify specific characteristics that impact individual performance [27-29]. A multiple baseline single subject design was used for this study so that investigators could determine effective parameters of a repetition-based training program for propulsion biomechanics. A training program that produces efficient propulsion skills is crucial for clinical rehabilitation in order to prevent or delay the onset of secondary injuries such as chronic for data collection and participant interaction was approved by the institutional review board at Washington University.

\section{Participants}

Five adults (three men, two women, aged $39.0 \pm 30$ ) with an SCI who use a manual wheelchair were recruited for this pilot investigation (Table 1). This study included participants with traumatic and non-traumatic SCI, those with incomplete and complete SCIs, and range of American spinal injury Association Impairment Scale (AIS) classifications (Table 1). Participants were recruited from a variety of resources in the St. Louis area that serve people with SCIs. Recruitment resources included local disability organizations, support groups, independent living centers, and rehabilitation hospitals. Participants were screened by phone to ensure that they met the following inclusion criteria: experienced an SCI of C6 or below in the past 36 months, had the ability to actively self-propel their own manual wheelchair using their upper extremities, used a manual wheelchair for at least $75 \%$ of daily activities, were between the ages of 18 and 69, understood spoken english as determined by a brief interview, and had the ability to provide informed consent to participate. All participants were screened for pain according to the Numeric Pain Scale (NPS) during the initial interview and at the beginning of each session to ensure that pain did not interfere with propulsion training [30]. Informed written consent was obtained from all participants prior to participation in this study.

\section{Training Program}

Participants completed a nine-session training program; each training session was 90 minutes long and included pre- and post-assessments, propulsion training, and skill training (Table 2). All participants completed at least one training session per week; a maximum of two training sessions per participant occurred in the period of one week. This training program utilized principles from motor learning theory to improve propulsion biomechanics. The overarching principle of completing task-specific repetitions was

\begin{tabular}{|c|c|c|c|c|c|c|c|c|c|}
\hline Participant & Gender & Dominant hand & Age & Months since injury & Traumatic SCI & Level of Injury & $\begin{array}{l}\text { Incomplete or } \\
\text { complete injury }\end{array}$ & ASIA level & Receiving therapy \\
\hline 1 & $\mathrm{~F}$ & $\mathrm{R}$ & 69 & 36 & No & Not reported & Incomplete & C & No \\
\hline 2 & $\mathrm{~F}$ & $R$ & 46 & 9 & Yes & $\mathrm{C} 6 / \mathrm{C} 7$ & Complete & A & Yes \\
\hline 3 & M & $R$ & 34 & 6 & Yes & T12 & Incomplete & B & No \\
\hline 4 & M & $R$ & 26 & 18 & Yes & T11 & Complete & A & Yes \\
\hline 5 & M & $\mathrm{R}$ & 20 & 11 & Yes & $\mathrm{C} 7$ & Incomplete & c & Yes \\
\hline
\end{tabular}

overuse injuries as well as to promote participation throughout the individual's lifespan. The purpose of this study was to determine the number of propulsion repetitions necessary to produce positive changes in propulsion biomechanics for new MWU with SCIs. This study aims to answer the following research questions: 1) Can repetition-based training be used to teach propulsion biomechanics in new MWU with SCI? 2) How much practice or how many repetitions does a new MWU with SCI need for motor learning in wheelchair propulsion to occur?

\section{Methods}

This study utilized a single subject design. Participants completed a nine-session manual wheelchair training program. The protocol accompanied by other principles from motor learning theory in order to produce improved motor cortical representations [31,32].

\begin{tabular}{|c|c|}
\hline Time (minutes) & Training Focus \\
\hline 0:00-15:00 & Check in, pre-session assessment, intro to training \\
\hline $15: 00-25: 00$ & Propulsion A (approx. 250-350 reps) \\
\hline $25: 00-45: 00$ & Wheelchair Skill 1 \\
\hline $45: 00-50: 00$ & Break \\
\hline $50: 00-60: 00$ & Propulsion B (approx. 250-350 reps) \\
\hline $60: 00-80: 00$ & Wheelchair Skill 2 \\
\hline 80:00-90:00 & Post-session assessment, wrap up \\
\hline \multicolumn{2}{|c|}{ Table 2: Example of timing of training session and repetitions achieved. } \\
\hline
\end{tabular}


Propulsion skills were taught for a total of 20 minutes each training session, divided into two propulsion sets of 10 minutes each. Propulsion Set A focused on cueing the participant to lengthen the amount of time his or her hand was in contact with the pushrim during the push phase of propulsion. During Propulsion Set B, participants were cued to drop their hand toward the wheel axle during the recovery phase of propulsion. Cues given during the propulsion sets were not scripted but were individualized for each participant as needed. The limited focus of each propulsion set utilized the motor learning concept of limiting the variables presented [33,34]. Researchers varied the order of the two propulsion sets throughout the training program to maximize random practice. Participants were coached throughout each set in order to correct propulsion form and to provide them with extrinsic post-responsive information, or external feedback, on propulsion movements [35].

In both animal and human studies, the reported amount of movement (or number of repetitions) required to acquire a skill varies [16]. In neurorehabilitation literature, recommendations for turning a movement into a learned skill range from 300-800 repetitions per rehabilitation session $[16,36,37]$. Therefore, each training session contained 500 to 700 total repetitions that were completed on the Wheel Mill System (WMS). The number of repetitions achieved increased every third session, as the clinicians determined that the participants were able to tolerate more (i.e., sessions 1-3 achieved 500 repetitions each, sessions 4-6 achieved 600 repetitions each, and sessions 7-9 achieved 700 repetitions each). To allow enough sessions for change to occur the training was structured for a total of nine sessions. A total of 5,400 repetitions were achieved after completion of all nine sessions. Each session also included training on skills such as transfer skills, maneuvering environmental barriers (eg: ramps and curbs), and gaining independence in using the manual chair. Wheelchair skills training acted as a strategy to vary the practice schedule and provide breaks from performing propulsion sets $[38,39]$.

\section{Assessment Tools}

\section{Wheelmill system}

A wheelchair dynamometer (roller based) system, the WMS, was used during the training sessions for data collection and training (Figure 1) [40]. The WMS is a computer-controlled dynamometer that can simulate various resistant surfaces and slopes in a laboratory or clinical setting. The WMS has a roller platform that simulates a variety of terrain types through the use of four independent motor-driven rollers. Users mount the WMS with their own chairs using their own wheels. The motors are controlled by the computer and move the rollers, allowing for different resistances and glides on each wheelchair wheel. The WMS is controlled by software written in Microsoft Visual C [41]. The software interface displays speed, slope, and distance. The WMS also has the ability to measure average and peak forces exerted on the wheel during propulsion. The WMS has similar force values as compared to the forces measured by an instrumented wheel [40].

\section{Microsoft Kinect}

The Microsoft Kinect sensor is a portable, inexpensive motion sensing device that can be used in conjunction with a personal computer to monitor the three-dimensional position and orientation of a person's body segments without the use of reflective markers or wearable devices [42]. The Microsoft Kinect was shown to have strong concurrent validity with multiple camera 3D video motion analysis software in a study on postural control [42]. During data collection,

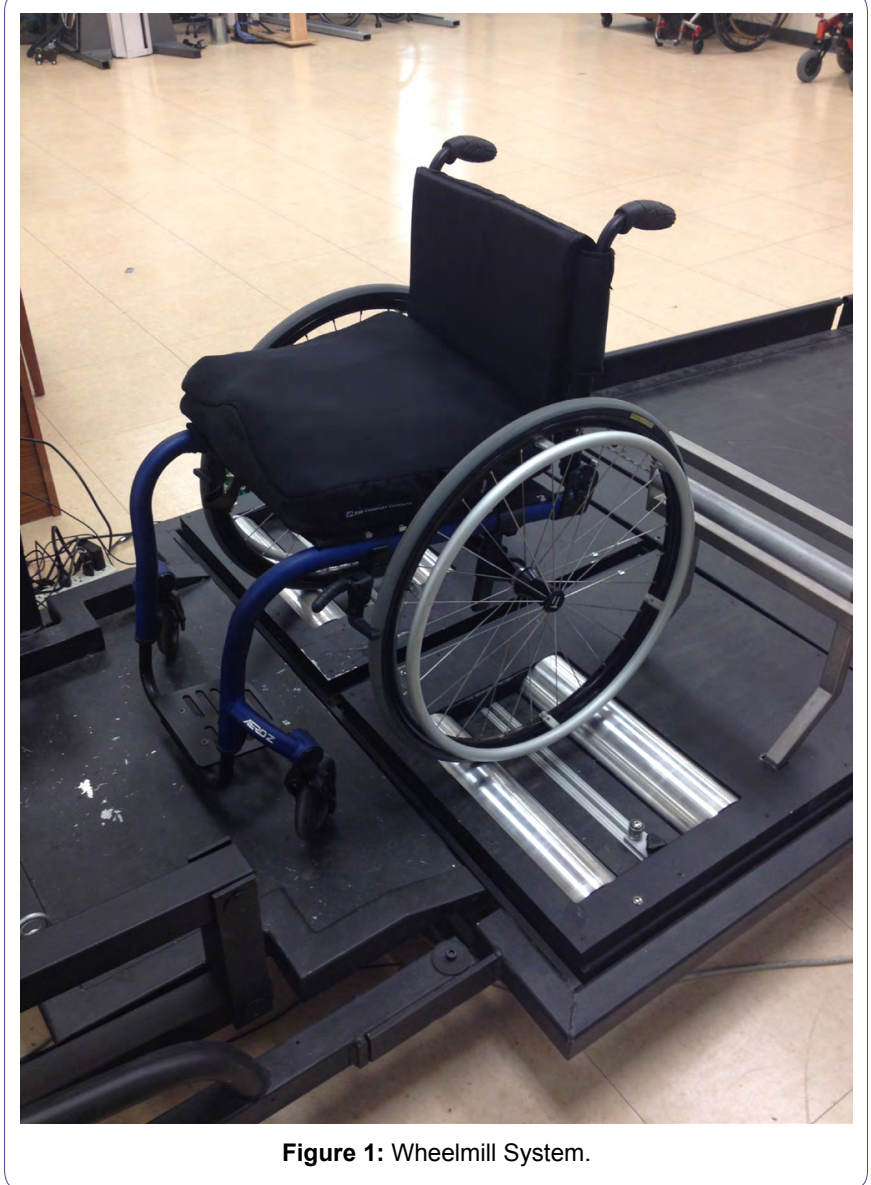

two laptop computers and two Kinect sensors were set up on either side of the participant and were used to measure the movement of the head, torso, and left and right upper extremities during wheelchair training.

\section{Dartfish}

Videos taken during the pre- and post-assessments were processed using Dartfish ProSuite 5.5 Video Software solutions [43]. Dartfish tracks kinematic movement in two-dimensional videos with a $\pm 5 \mathrm{~mm}$ accuracy compared to 3D tracking [43]. Two video cameras were used during data collection; they were positioned at the level of the right and left wheel axles, with the frame capturing the area from the bottom of the wheel to the participant's shoulder. Videos taken during the pre- and post-assessments were reworked using Dartfish ProSuite 5.5 Video Software Solutions, which tracks kinematic movement in two-dimensional videos [43].

\section{Assessment Procedures}

Participants completed assessments at the beginning and end of each training session to determine changes in propulsion biomechanics according to The CSCM clinical practice guidelines [8]. Assessments accounted for 20 minutes of the total session time; each pre- and post-assessment averaged eight minutes, with extra time added for technical issues and participant breaks. Changes in propulsion biomechanics include decreasing push forces (peak propulsion force, average force, force rate of rise), decreasing push cadence (number of pushes per second), increasing the push length 
(push angle, start angle), and dropping the hand toward the axle during recovery (push loop height).

\section{Establishing speeds}

During the first training session, each participant was fitted to the WMS to accommodate the custom aspects of his or her wheelchair. Once the WMS was adjusted for the individual participant, a decay value that simulated a smooth, flat surface was chosen [40]. Participants were asked to push on the WMS at a comfortable pace that felt like pushing casually across a smooth flat surface. Once a comfortable pace was achieved, the speed was recorded and used as the assessment speed for the remainder of the assessments, during which participants were asked to maintain this speed for a period of 30 seconds.

\section{Pre-session assessment}

At the beginning of each training session, data concerning the participants' push biomechanics were collected using the WMS, Microsoft Kinect, and video cameras. Participants rolled onto the WMS, and the wheel chair was secured. The Kinect systems were set up at the level of the wheel axle on both right and left wheels. Video cameras were set up at the level of the wheel axle on both the right and left wheels; the frame of the camera captured the image of the entire wheel and the participant's shoulder joint. The participants completed one minute of warm up pushes prior to data collection. The visual feedback monitor was angled toward the participants so they could monitor their speed and adjust it accordingly. Participants were asked to do two backwards pushes in order to synchronize the WMS, Kinect, and video cameras to the beginning of data collection. Following the two backward pushes, participants were asked to achieve their assessment speed by using cues from the visual feedback monitor; once a steady state was achieved, the participants were asked to maintain the speed for 30 seconds. Following the 30 seconds of data collection, the participants were asked to slow to a stop and rest for one minute; the WMS, Kinect, and video cameras were stopped and the data were saved. Participants completed three additional 30 -second assessments at various standardized speeds, with one-minute breaks between each speed; however, the data were not analyzed for this particular study.

\section{Post-session assessment}

During the last ten minutes of each training session, participants push biomechanics were assessed using the WMS, Microsoft Kinect, and video cameras. Post-training session assessment followed the protocol of pre-training session assessment.

\section{Data Processing}

Data were processed and analyzed from nine different assessment points throughout the training program. A baseline was established with data from the first four assessment points, prior to blocked repetition. The remaining processed data points were determined based on the number of repetitions achieved (Table 3 ) and will be referred to as "dosing levels" to differentiate between training sessions and assessment points. The dosing levels were defined by the number of repetitions achieved. From each dosing level, data from five consecutive pushes were selected and processed. The five consecutive pushes were selected once the participant had reached a steady state as determined by the speed output from the WMS data. Kinematic and kinetic measurements were taken at all dosing levels in order to determine participants' peak force, average force, force rate of rise, cadence, push angle, start angle, and push loop height (Table 4).

\begin{tabular}{|c|c|}
\hline Dosing Level & Repetitions Achieved \\
\hline Baseline & 0 \\
\hline Baseline & $<500$ \\
\hline Level 1 & 1000 \\
\hline Level 2 & 1500 \\
\hline Level 3 & 2100 \\
\hline Level 4 & 2700 \\
\hline Level 5 & 3300 \\
\hline Level 6 & 4000 \\
\hline Level 7 & 5400 \\
\hline Table 3: Dosing levels based on repetitions achieved.
\end{tabular}

\begin{tabular}{|c|c|c|}
\hline Outcome & Unit of Measurement & Assessment Measure \\
\hline Peak Force & Neutons (N) & WMS \\
\hline Average Force & $\mathrm{N}$ & WMS \\
\hline Force Rate of Rise & $\mathrm{N} / \mathrm{sec}$ & WMS \\
\hline Cadence & Pushes/sec & WMS \\
\hline Push Angle & Degrees & Dartfish \\
\hline Start Angle & Degrees & Dartfish \\
\hline Push Loop Height & Meters & Kinect \\
\hline \multicolumn{3}{|c}{ Table 4: Summary of outcomes. } \\
\hline
\end{tabular}

\section{Wheelmill system}

The Tangential Force $\left(\mathrm{F}_{\mathrm{T}}\right)$ from the WMS was calculated from the motor control signal controlling the speed of the rollers [40]. The torque applied to the rollers by the wheels of the wheelchair is sensed by the motor, and a control signal is calculated to turn the rollers at the appropriate speed. The $\mathrm{F}_{\mathrm{T}}$ is calculated by subtracting a speed-dependent voltage offset from this control signal and multiplying it by a conversion coefficient. The $\mathrm{F}_{\mathrm{T}}$ calculation was verified using the Smart Wheel (Three Rivers Holdings, LLC) as the gold standard [40]. The key kinetic variables that were calculated through the WMS were peak propulsion force, average propulsion force, and force rate of rise. Once the participant reached a steady state of speed, variables from the five consecutive propulsion strokes were processed. The motor control signal resulting from the torque applied to the rollers of the WMS was converted to $\mathrm{F}_{\mathrm{T}}$ through custom Microsoft Excel spreadsheets [44]. Peak $\mathrm{F}_{\mathrm{T}}$ was calculated as the average of the peak values achieved during the five propulsion strokes. Average $\mathrm{F}_{\mathrm{T}}$ was calculated as the average of $\mathrm{F}_{\mathrm{T}}$ values during the entire propulsion phase of the five strokes. Rate of rise of $\mathrm{F}_{\mathrm{T}}$ was calculated as Newton's divided by the number of seconds taken from the beginning of the propulsion phase to the peak value [2].

\section{Microsoft Kinect}

Depth data from the Kinect sensor were automatically converted into skeletal data using the skeleton tracking algorithm built into the Microsoft Kinect Software Development Kit (SDK). Each skeletal frame was streamed into a custom-written program (C\#) and saved in an output file for post-processing. Three-dimensional skeletal data from the Kinect sensor were post-processed within MATLAB [45]. The straight-line distance from the position of the hand marker to the origin of the Kinect reference frame was calculated and used to divide the movement data into push cycles. 
Push loop height was defined as the straight-line distance between the hand marker at its maximum and minimum vertical positions measured in the reference frame of the Kinect sensor (Figure 2). Pushes were time-synchronized between the WMS and Kinect data by counting the number of full push cycles and aligning the first and last cycles.

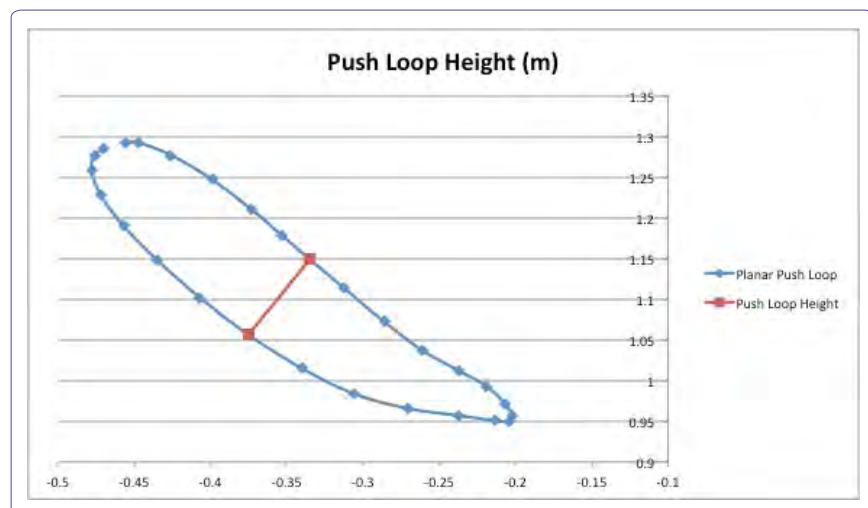

Figure 2: Illustration of Kinect output data and push loop height variable.

\section{Dartfish}

Videos were uploaded to Dartfish ProSuite 5.5; the five pushes identified for data analysis were time-synchronized to the first push of the WMS and Kinect based on visual analysis. Each of the five pushes was processed to determine the increase in the participant's push length by measuring push angle and start angle. Data were processed using Dartfish's angle tracking tool. The beginning of the start angle was defined as when the third metacarpal first made contact with the pushrim, the end of the start angle was defined as when the third metacarpal was at the top dead center of the pushrim, and the angle was measured in relation to the wheel axle. The push angle was determined as the beginning of the push when the third metacarpal first made contact with the pushrim until the hand left the pushrim, and the angle was measured as the relation of the two points to the wheel axle. The protocol for determining the push angle was based on preliminary validation of Dartfish video motion analysis with the gold standard kinematic measurement of the video motion capture system [46].

\section{Data Analysis}

A single subject design was used to determine changes in propulsion biomechanics for each participant. Data were analyzed using a visual inspection method referred to as the percentage of non-overlapping data $[47,48]$. The baseline and intervention points were plotted. A criterion of stability was established for the baseline points based on the method of analysis; the percentage of difference between baseline points could not exceed the percentage of difference between baseline to intervention points. For variables we wanted to increase (push angle, start angle push loop height) the highest baseline point was identified, and the proportion of intervention points that exceeded (non-overlapping) the highest baseline point were calculated to determine the effective percentage (Figure 3; >90\% highly effective, $70-90 \%$ moderately effective, $50-70 \%$ minimally effective, $<50 \%$ ineffective) [47,48]. For variables we wanted to decrease (peak force, average force, force rate of rise, cadence), the lowest baseline point was identified and the porportion of intervention points that fell beneath that point were calculated. The dose level where change occurred was operationaly defined when three consecutive points fell above or below the percentage of overlapping data points.

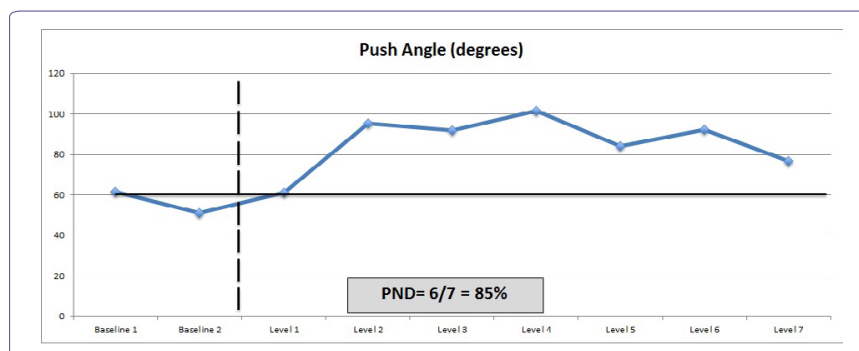

Figure 3: Example of analysis of percentage of overlapping data (PND) method.

\section{Result}

Each of the five participants had improvements in propulsion biomechanics, which occurred in the first dosing level (between 1000 and 2700 repetitions). There were variances in type of change in propulsion biomechanics among the participants. The effectiveness of change for each variable of each participant is outlined in table 5.

\section{Cadence}

Two of the participants demonstrated effective decreases in cadence during the training program. Participant 1 had a moderate effective decrease in cadence at dosing level 2, which was retained throughout the remainder of the training. Participant 4 demonstrated a minimally effective decrease at dosing level 1 , which was not retained at the completion of the training.

Kinetic variables

Two of the five participants had a decrease in peak propulsion force. Participant 3 had a highly effective decrease in peak propulsion force that occurred at dosing level 1 (1000 repetitions) and continued to decrease with higher dosing levels. Participant 5 had a minimally effective decrease in peak propulsion force at dosing level 2 (1500 repetitions), which was seen at dosing levels 3 and 6 but was not retained at the end of the training. Average propulsion force decreased for three of the five participants. Participant 3 had a highly effective decrease in average propulsion force at dosing level 1 that steadily decreased with additional repetitions. Participant 1 had a minimally effective decrease in average propulsion force at dosing level 4 (2700 repetitions) that was retained to the end of the training. Force rate of rise decreased for two of the five participants. Participant 1 demonstrated highly effective decrease in force rate of rise at level 1 , which was maintained with higher dosing levels. Participant 5 had a moderate effective decrease at dosing level 2 , with a decrease in force rate of rise seen at dosing levels 3, 4, 6 and 7. Kinetic data is not reported for Participant 2 due to missing baseline data and, thus, inconclusive results.

\section{Kinematic variables}

Three participants had an effective increase in push angle during the training program. Participant 1 demonstrated a moderate effective increase at dosing level 2, meaning that the push angle increased following 1500 repetitions and the increase was maintained throughout the remainder of the dosing levels. Participant 3 demonstrated a moderate effective increase of push angle at dosing level 1 that persisted through dosing level 6 but decreased at dosing level 7. Participant 5 had a highly effective increase in push angle at dosing level 1 that was retained through the remainder of the dosing 
Citation: Will K, Engsberg JR, Foreman M, Klaesner J, Birkenmeier R, et al. (2015) Repetition-Based Training for Efficient Propulsion in New Manual Wheelchair Users. J Phys Med Rehabil Disabil 1: 001.

- Page 6 of 9 •

\begin{tabular}{|c|c|c|c|c|c|c|c|c|c|c|}
\hline \multirow[b]{2}{*}{ Variable } & \multicolumn{2}{|c|}{ Participant 1} & \multicolumn{2}{|c|}{ Participant 2} & \multicolumn{2}{|c|}{ Participant 3} & \multicolumn{2}{|c|}{ Participant 4} & \multicolumn{2}{|c|}{ Participant 5} \\
\hline & Change & Dosing level & Change & Dosing level & Change & Dosing level & Change & Dosing level & Change & Dosing level \\
\hline Peak force & - & - & NR & NR & $+++^{*}$ & 1 & - & - & $+^{*}$ & 2 \\
\hline Rate of rise & $+++^{*}$ & 1 & NR & NR & - & - & - & - & $++^{*}$ & 2 \\
\hline Cadence & $++^{*}$ & 2 & - & - & - & - & $+^{*}$ & 1 & - & - \\
\hline Push angle & $++^{*}$ & 2 & ++ & 1 & $++^{*}$ & 1 & - & - & $+++^{*}$ & 1 \\
\hline Start angle & $+++^{*}$ & 1 & ++ & 1 & $++^{*}$ & 1 & - & - & - & - \\
\hline Push loop height & $++^{*}$ & 2 & - & - & $++^{*}$ & 1 & - & - & - & - \\
\hline
\end{tabular}

Table 5: Variable change at dosing level for each participant.

NR: not reported; + minimally effective change $(50-70 \%) ;++$ moderately effective change $(70-90 \%) ;+++$ highly effective change $(>90 \%) ;-$ no change; * change indicates improvement in variable

levels. Participant 1 demonstrated a highly effective increase in start angle at dosing level 1 that was retained at following dosing levels. A moderate effective increase in start angle was seen in Participant 3 at dosing level 1, with the increase retained through dosing level 6 but not seen at dosing level 7 . Three of the five participants had a moderate effective increase in push loop height. Participant 1 demonstrated this change at dosing level 2, and Participant 4 demonstrated this change at dosing level 3; the changes were retained throughout the training for both participants. Participant 3 demonstrated the increase in push loop height at dosing level 1 , and retention was seen in all other dosing levels other than dosing level 5. Participant 2 had a moderate effect in decrease of push angle and start angle at dosing level 1 that was seen in dosing levels 2 and 3 and 5-7.

\section{Discussion}

The purpose of this pilot study was to estimate the number of repetitions necessary to produce changes in propulsion biomechanics of MWU. Motor learning theory states that one must perform hundreds of task-specific repetitions over several sessions in order for a movement to be learned and retained $[14,17,18,49]$. The number of repetitions necessary to produce change in propulsion biomechanics of this population has not been a focus of research until this study. The participants in this study improved propulsion biomechanics following task-specific repetition training but the response to the intervention varied among individuals. Among all five of the participants there was variability between the type of change in propulsion biomechanics and the corresponding dose level at which change occurred. The majority of changes that occurred during the training program happened during the first four levels of repetition dosing. All participants displayed changes in propulsion biomechanics at the first dosing level, following 1000 task-specific repetitions. It is unclear whether these changes would have been retained throughout the remainder of the intervention if the training were stopped. Long-term retention of the changes was also not addressed in this pilot study and should be considered in future studies. Further, the effectiveness of change that occurred at later dosing levels may have been limited due to the method of visual analysis used. In addition, it is difficult to determine how baseline proficiency impacted the individual's ability to change over subsequent training sessions.

This study had many methodological limitations, including the variability of injury levels among participants, the small sample size, and restricted baseline measurements. Due to health, financial, and logistical complications following SCI, recruitment of participants was challenging and led to the small sample size. In addition, the inclusion criteria were expanded to include a participant who was 36 months post-SCI; although the participant was not newly injured prior to the training program a family member pushed her in her wheelchair throughout the day. This participant was therefore considered a new independent MWU. This study was limited by logistical difficulties with participant schedules. All participants completed at least one training session per week; however, the exact number of days between each training session was not controlled for and, thus, is a limitation of this study. Difficulty in scheduling the multitude of training sessions for each participant restricted the number of baseline measurements and limits the stability of the results. However, the single subject design methodology allowed for the identification of characteristics relevant to individual performance and will be useful in developing larger studies [27-29].

This study aligns with other literature, which indicates that improvements in propulsion biomechanics can be made in new MWU [12] and that wheelchair positioning $[8,50,51]$ and level of injury [50-52] may play an important role in propulsion biomechanics. The differences in type of biomechanical change among participants may be explained by differences in wheelchair positioning and level of injury. Chair positioning plays an important role in propulsion efficiency and may explain variances in biomechanical change among participants. Elbow angle is directly related to the height of the seat in relation to the wheels of the chair. A lower seat corresponds with a smaller elbow angle and is associated with increased motion of the upper extremity [50,51], increased push angle [2], and reduced cadence [51]. The CSCM recommends that the angle of the elbow when the hand is at the top dead center of the pushrim during propulsion be between 100 and 120 degrees [8]. Four of the five participants were within a reasonable range of the recommended elbow angle; however, only two demonstrated an increased push angle, and only one had decreased cadence (Table 6). The CSCM states that an elbow angle below $100^{\circ}$, as was the case with Participant 1 from our study, may increase the likelihood of shoulder impingement due to adducted push $[2,8,51]$. The slightly larger elbow angle in Participants $2\left(122^{\circ}\right)$ and $5\left(122^{\circ}\right)$ may have impacted their ability to drop their hands down below the pushrim during recovery to produce an effective change in increased push loop height. Due to transferring circumstances, the seat height for two participants could not be lowered or their ability to transfer in and out of their chair would have been impacted. Lowering the seat height may have helped improve propulsion biomechanics for Participant 2 and Participant 5. Only one participant whose elbow angle was within the recommended range had a decreased cadence as a result of the training. Seat positioning may explain the decrement in propulsion kinematics for Participant 2, 
as the seat angle was decreased following the baseline measurements to increase stability in the chair. By closing the seat-to-back angle, the participant was positioned to start the push phase propulsion farther forward on the pushrim than before, decreasing the start and push angles. Change in elbow angle between sessions was found to be ineffective for all five participants and, therefore, it is unlikely that the improvements in propulsion biomechanics are a result of chair positioning alone. Task-specific repetition training in conjunction with the wheelchair positioning that best facilitates function in tasks including propulsion and transferring may provide the most beneficial treatment in prolonging the health of the upper extremities for new MWU.

\begin{tabular}{|c|c|}
\hline Participant & ${\text { Elbow } \text { Angle }^{*}}^{*}$ \\
\hline 1 & 85.24 \\
\hline 2 & 122.29 \\
\hline 3 & 119.2 \\
\hline 4 & 117.3 \\
\hline 5 & 122.3 \\
\hline \multicolumn{2}{|c|}{ Table 6: Participant elbow angle. } \\
\hline
\end{tabular}

Propulsion biomechanics are greatly influenced by the level of SCI, as muscle innervation of the upper extremities decreases with higher-level injuries. Participants with tetraplegia, such as Participants 2 and 5, have been shown to have a less efficient push due to lack of functioning of the wrist, hand, and triceps muscles [52-54]. Lack of hand functioning requires increased force applied inward on the pushrim [52] as well as increased wrist extension [55] in order to maintain contact during the push phase of propulsion. Participant 5 (C7 tetraplegia), displayed an increased push angle with a less effective decreased peak force and force rate of rise. In this case, the increased push angle may have directly influenced the peak force and force rate of rise, as the CSCM states that when forces are applied in longer strokes, the efficiency increases without amplified forces on the upper extremity [8]. The three participants classified with paraplegia had full innervation of the upper extremities, which allowed them the opportunity to make more improvements in their propulsion biomechanics. Both Participants 1 and 3 had innervation of triceps brachii muscles and, thus, the ability to extend their elbows during the recovery phase of propulsion, which increased the length of the push as displayed by an increased start angle as well as an increased push angle. Participants 1 and 3 also had an increased push loop height, which may be attributed in part to their ability to extend their elbows fully during the recovery phase of propulsion. This study agrees with current literature that propulsion biomechanics are impacted by level of SCI [52-55]. We did not assess upper extremity strength at the beginning or the end of the study, which may be a limitation to understanding how muscle innervation and strength plays a role in propulsion training. Future studies should consider the differences in propulsion biomechanics among participants with varied levels of SCI while using task-specific repetitions to improve propulsion biomechanics.

This pilot study had both strengths and limitations in the methods of data collection due to the innovative nature of the equipment used. The Microsoft Kinect system was able to measure three-dimensional kinematic movement of the hand. However, all calculations using data from the Kinect sensor were performed in the Kinect reference frame due to the fact that only skeletal data was collected. No data relative to the position of the wheelchair was obtained using the Kinect and, therefore, coordinate transformations from the Kinect reference frame to the wheelchair or global reference frames were impossible. Further research should be done to determine the feasibility of the use of the Microsoft Kinect for the clinical kinematic measurement of manual wheelchair propulsion. Strength of this study was the innovative WMS, which allowed kinematic data to be collected while preserving the natural wheelchair positioning and setup for participants, allowing for more accurate data to be collected.

This pilot study is consistent with other literature in its findings that wheelchair positioning [50,51] and level of SCI [52-55] affect propulsion biomechanics. Further, this study adds to the literature on the importance and feasibility of efficient propulsion biomechanics for new MWU with SCIs.

\section{Conclusion}

This pilot study demonstrates the ability of new MWU to make improvements in propulsion biomechanics following task-based repetitions. Researchers aimed to improve propulsion biomechanics by following the guidelines established by the CSCM by decreasing forces applied to the pushrim (push force), increasing the length of propulsion (push angle), decreasing overall propulsion cadence, and dropping the hand down toward the axle during the recovery phase of propulsion (increased push loop height). All participants in this study displayed improvements in at least one outcome. The variability among participants in the type of change that occurred and the dosing level at which change occurred demonstrates the variability among individuals with SCIs. More research is needed to determine the appropriate dose required for retained change as well as the impact that chair positioning and level of injury have on motor learning.

\section{Acknowledgements}

This study was funded by the Missouri Spinal Cord Injuries Research Program. The authors would like to thank the participants who took the time to participate in this project.

\section{References}

1. Akbar M, Balean G, Brunner M, Seyler TM, Bruckner T, et al. (2010) Prevalence of rotator cuff tear in paraplegic patients compared with controls. J Bone Joint Surg Am 92: 23-30.

2. Boninger ML, Baldwin M, Cooper RA, Koontz A, Chan L (2000) Manual wheelchair pushrim biomechanics and axle position. Arch Phys Med Rehabil 81: 608-613.

3. Collinger JL, Impink BG, Ozawa H, Boninger ML (2010) Effect of an intense wheelchair propulsion task on quantitative ultrasound of shoulder tendons. PM R 2: 920-925.

4. Nyland J, Quigley P, Huang C, Lloyd J, Harrow J, et al. (2000) Preserving transfer independence among individuals with spinal cord injury. Spinal Cord 38: 649-657.

5. Robertson RN, Boninger ML, Cooper RA, Shimada SD (1996) Pushrim forces and joint kinetics during wheelchair propulsion. Arch Phys Med Rehabil 77: 856-864.

6. Rice I, Gagnon D, Gallagher J, Boninger M (2010) Hand rim wheelchair propulsion training using biomechanical real-time visual feedback based on motor learning theory principles. J Spinal Cord Med 33: 33-42.

7. Koontz AM, Cooper RA, Boninger ML, Yang Y, Impink BG, et al. (2005) A kinetic analysis of manual wheelchair propulsion during start-up on select indoor and outdoor surfaces. J Rehabil Res Dev 42: 447-458. 
Citation: Will K, Engsberg JR, Foreman M, Klaesner J, Birkenmeier R, et al. (2015) Repetition-Based Training for Efficient Propulsion in New Manual Wheelchair Users. J Phys Med Rehabil Disabil 1: 001.

8. Paralyzed Veterans of America Consortium for Spinal Cord Medicine (2005) Preservation of upper limb function following spinal cord injury: a clinical practice guideline for health-care professionals. J Spinal Cord Med 28: 434-470.

9. Boninger ML, Souza AL, Cooper RA, Fitzgerald SG, Koontz AM, et al. (2002) Propulsion patterns and pushrim biomechanics in manual wheelchair propulsion. Arch Phys Med Rehabil 83: 718-723.

10. Routhier F, Vincent C, Desrosiers J, Nadeau S (2003) Mobility of wheelchai users: a proposed performance assessment framework. Disabil Rehabil 25 : 19-34.

11. Rodgers MM, Keyser RE, Rasch EK, Gorman PH, Russell PJ (2001) Influence of training on biomechanics of wheelchair propulsion. J Rehabil Res Dev 38: 505-511.

12. Rice LA, Smith I, Kelleher AR, Greenwald K, Boninger ML (2014) Impact of a wheelchair education protocol based on practice guidelines for preservation of upper-limb function: a randomized trial. Arch Phys Med Rehabil 95: 10-19.

13. Lang CE, MacDonald JR, Gnip C (2007) Counting repetitions: an observational study of outpatient therapy for people with hemiparesis post-stroke. $J$ Neurol Phys Ther 31: 3-10.

14. Boudreau SA, Farina D, Falla D (2010) The role of motor learning and neuroplasticity in designing rehabilitation approaches for musculoskeletal pain disorders. Man Ther 15: 410-414.

15. Dayan E, Cohen LG (2011) Neuroplasticity subserving motor skill learning Neuron 72: 443-454.

16. Lang CE, Macdonald JR, Reisman DS, Boyd L, Jacobson Kimberley T, et al (2009) Observation of amounts of movement practice provided during stroke rehabilitation. Arch Phys Med Rehabil 90: 1692-1698.

17. Nudo RJ (2006) Mechanisms for recovery of motor function following cortical damage. Curr Opin Neurobiol 16: 638-644.

18. Karni A, Meyer G, Rey-Hipolito C, Jezzard P, Adams MM, et al. (1998) The acquisition of skilled motor performance: fast and slow experience-driven changes in primary motor cortex. Proc Natl Acad Sci USA 95: 861-868.

19. Krebs HI, Hogan N, Hening W, Adamovich SV, Poizner H (2001) Procedural motor learning in Parkinson's disease. Exp Brain Res 141: 425-437.

20. Mak MK, Hui-Chan CW (2008) Cued task-specific training is better than exercise in improving sit-to-stand in patients with Parkinson's disease: A randomized controlled trial. Mov Disord 23: 501-509.

21. Girgis J, Merrett D, Kirkland S, Metz GA, Verge V, et al. (2007) Reaching training in rats with spinal cord injury promotes plasticity and task specific recovery. Brain 130: 2993-3003.

22. Lovely RG, Gregor RJ, Roy RR, Edgerton VR (1986) Effects of training on the recovery of full-weight-bearing stepping in the adult spinal cat. Exp Neurol 92: 421-435.

23. Behrman AL, Harkema SJ (2000) Locomotor training after human spinal cord injury: a series of case studies. Phys Ther 80: 688-700

24. Wirz M, Zemon DH, Rupp R, Scheel A, Colombo G, et al. (2005) Effectiveness of automated locomotor training in patients with chronic incomplete spinal cord injury: a multicenter trial. Arch Phys Med Rehabil 86: 672-680.

25. Hou JM, Yan RB, Xiang ZM, Zhang H, Liu J, et al. (2014) Brain sensorimotor system atrophy during the early stage of spinal cord injury in humans. Neuroscience 266: 208-215.

26. Bruehlmeier M, Dietz V, Leenders KL, Roelcke U, Missimer J, et al. (1998) How does the human brain deal with a spinal cord injury? Eur J Neurosci 10 3918-3922.

27. Graham JE, Karmarkar AM, Ottenbacher KJ (2012) Small sample research designs for evidence-based rehabilitation: issues and methods. Arch Phys Med Rehabil 93: 111-116.

28. Zhan S, Ottenbacher KJ (2001) Single subject research designs for disability research. Disabil Rehabil 23: 1-8.
29. Bobrovitz CD, Ottenbacher KJ (1998) Comparison of visual inspection and statistical analysis of single-subject data in rehabilitation research. Am J Phys Med Rehabil 77: 94-102.

30. Ferreira-Valente MA, Pais-Ribeiro JL, Jensen MP (2011) Validity of four pain intensity rating scales. Pain 152: 2399-2404.

31. Hubbard IJ, Parsons MW, Neilson C, Carey LM (2009) Task-specific training: evidence for and translation to clinical practice. Occup Ther Int 16: 175-189.

32. Bayona NA, Bitensky J, Salter K, Teasell R (2005) The role of task-specific training in rehabilitation therapies. Top Stroke Rehabil 12: 58-65.

33. McEvoy LK, Smith ME, Gevins A (1998) Dynamic cortical networks of verbal and spatial working memory: effects of memory load and task practice. Cereb Cortex 8: 563-574.

34. Schmidt RA, Wulf G (1997) Continuous concurrent feedback degrades skill learning: implications for training and simulation. Hum Factors 39: 509-525.

35. Schmidt RA, Young DE, Swinnen S, Shapiro DC (1989) Summary knowledge of results for skill acquisition: support for the guidance hypothesis. J Exp Psychol Learn Mem Cogn 15: 352-359.

36. Birkenmeier RL, Prager EM, Lang CE (2010) Translating animal doses of task-specific training to people with chronic stroke in 1-hour therapy sessions: a proof-of-concept study. Neurorehabil Neural Repair 24: 620-635.

37. Kimberley TJ, Samargia S, Moore LG, Shakya JK, Lang CE (2010) Comparison of amounts and types of practice during rehabilitation for traumatic brain injury and stroke. J Rehabil Res Dev 47: 851-862.

38. Shea JB, Morgan RL (1979) Contextual interference effects on the acquisition, retention, and transfer of a motor skill. J Exp Psychol Learn Mem Cogn 5: $179-187$

39. Rey PD, Whitehurst M, Wughalter E, Barnwell J (1983) Contextual interference and experience in acquisition and transfer. Percept Motor Skill 57: 241 242

40. Klaesner J, Morgan KA, Gray DB (2014) The development of an instrumented wheelchair propulsion testing and training device. Assist Technol 26: 24-32.

41. Microsoft (2015) The latest supported Visual C++ downloads. Microsoft Visual C studios, USA.

42. Clark RA, Pua YH, Fortin K, Ritchie C, Webster KE, et al. (2012) Validity of the Microsoft Kinect for assessment of postural control. Gait Posture 36: 372-377.

43. Eltoukhy M, Asfour S, Thompson C, Latta L (2012) Evaluation of the performance of digital video analysis of human motion: Dartfish tracking system. Int J Sci Eng Res 3: 1-6.

44. Microsoft (2011) Microsoft Excel [computer software]. Microsoft, Redmond Washington, USA.

45. MATLAB Central (2010) MATLAB xUnit: new version available. MATLAB version 7.10.0, The MathWorks Inc., Natick, Massachusetts, USA.

46. Motion Analysis Corporation (2008) Cortex Version 1.0 Users Manual. Motion Analysis Corporation, Santa Rosa, CA, USA

47. Nye C, Turner H (2007) Conducting systematic reviews of randomized and non-randomized studies to inform evidence-based practice and policy: systematic reviews for single subject design. NCDDR's WORKSHOP for NIDRR Grantees. National Center for the Dissemination of Disability Research, USA

48. Scruggs TE, Mastropieri MA, Casto G (1987) The quantitative synthesis of single-subject research methodology and validation. Rem Spec Educ 8: 24-

49. Schmidt RA, Bjork RA (1992) New conceptualizations of practice: common principles in three paradigms suggest new concepts for training. Psychol Sci 3: 207-217.

50. Hughes CJ, Weimar WH, Sheth PN, Brubaker CE (1992) Biomechanics of wheelchair propulsion as a function of seat position and user-to-chair interface. Arch Phys Med Rehabil 73: 263-269. 
51. van der Woude LHV, Sargeant AJ (1989) Wheelchair propulsion: effect of seat height: 575. Med Sci Sport Exer 21: 96.

52. Kulig K, Newsam CJ, Mulroy SJ, Rao S, Gronley JK, et al. (2001) The effect of level of spinal cord injury on shoulder joint kinetics during manual wheelchair propulsion. Clin Biomech (Bristol, Avon) 16: 744-751.

53. Dallmeijer AJ, van der Woude LH, Veeger HE, Hollander AP (1998) Effectiveness of force application in manual wheelchair propulsion in persons with spinal cord injuries. Am J Phys Med Rehabil 77: 213-221.
54. Gil-Agudo A, Del Ama-Espinosa A, Pérez-Rizo E, Pérez-Nombela S, Pablo Rodríguez-Rodríguez $L$ (2010) Upper limb joint kinetics during manual wheelchair propulsion in patients with different levels of spinal cord injury. $J$ Biomech 43: 2508-2515.

55. Newsam CJ, Rao SS, Mulroy SJ, Gronley JK, Bontrager EL, et al. (1999) Three dimensional upper extremity motion during manual wheelchair propulsion in men with different levels of spinal cord injury. Gait Posture 10: 223-232. 